\title{
Contexte culturel et innovation pédagogique
}

\section{Olivier Sturge-Moore}

\section{OpenEdition}

Journals

Édition électronique

URL : http://journals.openedition.org/asp/3571

DOI : $10.4000 /$ asp.3571

ISSN : 2108-6354

\section{Éditeur}

Groupe d'étude et de recherche en anglais de spécialité

\section{Édition imprimée}

Date de publication : 1 décembre 1996

Pagination : 287-294

ISSN : 1246-8185

\section{Référence électronique}

Olivier Sturge-Moore, «Contexte culturel et innovation pédagogique», ASp [En ligne], 11-14 | 1996, mis en ligne le 09 mai 2013, consulté le 10 décembre 2020. URL : http://journals.openedition.org/asp/ 3571 ; DOI : https://doi.org/10.4000/asp.3571

Ce document a été généré automatiquement le 10 décembre 2020.

Tous droits réservés 


\title{
Contexte culturel et innovation pédagogique
}

\author{
Olivier Sturge-Moore
}

1 Le présent article soulèvera sans doute davantage de questions qu'il apportera de réponses. En effet, le vaste domaine de l'innovation pédagogique ouvre, par définition, un champ illimité de potentialités, nous suggérant d'emblée que toute nouvelle proposition pédagogique n'a rien de définitif et sera vraisemblablement rapidement dépassée.

\section{Tentatives de définitions}

2 Je voudrais tout d'abord tenter de définir la notion de contexte culturel. La plupart des recherches entreprises sur ce sujet concernent soit l'enseignement secondaire pour la langue générale, soit la littérature et la civilisation pour l'enseignement supérieur. En revanche, les perspectives semblent relativement nouvelles en langue de spécialité, pour autant que je sache. Voyons tout d'abord ce que l'on entend par la notion de contexte, essentiellement liée au développement de la pragmatique, parallèlement à celle "d'acte de parole » (c'est-à-dire d'interaction, de transaction...), de "performance», ou plus précisément de l'accomplissement de l'acte en contexte. Quant au troisième aspect, c'està-dire la notion de contexte, F. Armengaud le définit ainsi :

On entend par contexte la situation concrète où des propos sont émis, ou proférés, le lieu, le temps, l'identité des locuteurs, etc., tout ce que l'on a besoin de savoir pour comprendre et évaluer ce qui est dit. On s'aperçoit combien le contexte est indispensable lorsqu'on en est privé, par exemple lorsque des propos vous sont rapportés par un tiers, à l'état isolé; ils deviennent en général ambigus, inappréciables. Inversement le langage scientifique mais aussi le langage juridique se sont toujours efforcés de faire passer dans leurs propos - qui sont le plus souvent des textes écrits - toutes les informations contextuelles nécessaires à la bonne compréhension de ce qui est formulé. 

que beaucoup de documents pédagogiques, authentiques ou non, ne sont pas présentés, ou pas suffisamment, dans leur contexte.

4 F. Armengaud a établi une typologie des contextes: elle distingue quatre grandes catégories dont voici un résumé très succinct :

1. Le contexte circonstanciel, celui du monde réel

2. Le contexte présuppositionnel, constitué de tout ce qui est présumé par les interlocuteurs, leurs croyances, leurs attentes, leurs intentions, etc.

3. Le contexte interactionnel, celui de l'enchaînement des actes de langage, qui, dans une certaine mesure, peut être celui du cours de langue et de son déroulement.

4. Le contexte situationnel, qui suppose une culture partagée, socialement reconnue. C'est celui qui nous intéresse ici, notamment en ce qui concerne les actes illocutionnaires plus ou moins institutionnalisés, comme l'a montré Austin.

$$
\text { foisonnement des contextes risque vite d'apparaître comme inextricable. }
$$

va de même pour l'aspect culturel du contexte, ce qui rend insatisfaisante ma première question sur une possible définition; ou disons que toute tentative de définition sera obligatoirement réductrice. Cependant, de toute évidence, il s'agit d'une approche globale, prenant en compte tout ou tout au moins un maximum d'éléments exploitables pédagogiquement. Il est regrettable de laisser de côté des éléments qui sont exploitables.

7 Rappelons-nous la définition qu'en donne M.A.K. Halliday (1978: 5): «The total environment in which a text unfolds». Si nous sommes conscients de la possibilité de l'approche globale d'un document, le côté foisonnant et inextricable devrait normalement s'atténuer. Avant de poursuivre, il m'a paru nécessaire de tenter de classifier, de manière très schématique les différents domaines des langues de spécialité, en rapport avec la pertinence de leur contexte culturel, de manière à déterminer un degré de contextualisation.

1. Les langues de spécialité à caractère culturel (context-embedded language) telles que le droit (probablement le meilleur représentant). La langue de spécialité est ici indissociable de son contexte culturel. Elle utilisera " a high ratio of contextual information »; je reprends ici les termes de Cummins (1983).

9 2. La langue de spécialité à caractère scientifique, allant de la langue générale au vocabulaire général à orientation scientifique (VGOS) pour déboucher sur une véritable langue de spécialité (Baylon 1994 : 330).

10 3. Les langues scientifiques qui utiliseront: «a low ratio of contextual information », mais ceci dépendra également du genre employé (voir Swales 1990), ainsi que du registre, etc., la hiérarchie interne d'une communauté scientifique formant elle-même un contexte culturel et socio-professionnel. Selon les cas, elles peuvent être décrites comme utilisant " a context-reduced language ».

11 4. Les langues de spécialité techniques. Ici, il devient beaucoup plus difficile, voire impossible dans certains cas (une notice d'emploi pour un appareil, par exemple) de situer un document dans un contexte culturel de manière à pouvoir l'exploiter pédagogiquement. On peut alors parler, toujours avec Swales, de "context-free language " ou tout au moins d'un « very low ratio of contextual information ». 
12 Mais le genre utilisé peut laisser transparaître des traits culturels comme le fait remarquer Thomas Miller (1994). Cette remarque sur le genre nous entraîne un peu en dehors de notre sujet, mais je l'ai incluse car elle indique clairement que notre approche pédagogique en langue de spécialité doit être beaucoup plus précise qu'auparavant. Ceci est également valable pour l'exploitation pédagogique du contexte culturel. Cependant, il reste indéniable que plus un domaine est spécialisé sur le plan scientifique et encore plus dans les domaines techniques, plus le contexte culturel risque de s'appauvrir, laissant l'enseignant devant un véritable problème d'exploitation pédagogique. De manière générale, les contenus des enseignements en langue de spécialité sont calqués, après une analyse des besoins, sur la réalité et les exigences du monde professionnel du domaine concerné. Mais la relation entre texte et contexte est souvent insuffisamment élaborée, voire erronée ou même inexistante. Paul Gochet attire notre attention sur ce sujet :

L'idée qu'il existe un processus accumulatif par lequel on sollicite sans cesse de nouvelles coordonnées repose sur l'idée que l'information textuelle et l'information contextuelle sont deux sortes d'informations indépendantes qui s'additionnent pour donner la signification globale. Or cette idée est fausse. La portion pertinente du contexte n'est pas indépendante du texte. (cité dans Armengaud 1985 :58)

Texte et contexte sont si étroitement liés dans la réalité que nous devons en tirer des conclusions sur le plan pédagogique. Les professeurs de langue pensent peut-être trop souvent que le contexte est quelque chose d'acquis, de partagé entre les apprenants, surtout dans des classes qui ne sont pas particulièrement multiculturelles. Claire Kramsch (1993 : 78) va jusqu'à préconiser un enseignement spécifique du contexte :

Foreign language teachers should try and shape the instructional context in more natural ways. This points to the necessity of teaching context itself explicitly, and not assuming that it is transparent and agreed upon by everyone in the class

Si nous voulons aborder l'enseignement du contexte culturel, nous devons tout d'abord nous interroger sur leurs caractéristiques, voir jusqu'à quel point ils sont exploitables sur le plan pédagogique de manière à procéder à une analyse contextuelle. C'est ce que recommande également $\mathrm{H}$. Trocmé-Fabre quand elle écrit que :

la pratique pédagogique ferait un bon en avant si les formateurs concevaient leurs interventions dans une recherche, une perspective et une analyse contextuelle spatiale et temporelle. $(1987: 234)$

Il serait même souhaitable, mais c'est peut-être ambitieux, que ce soit les étudiants euxmêmes qui prennent en charge cette analyse contextuelle et déterminent un degré de contextualisation du document. Mais ce travail ne paraît possible que dans les cas où le contexte culturel est suffisamment clairement défini. Ils devront préciser les aspects culturels du contexte étudié, ses fonctions référentielles, ses connotations et implicites, etc. Bien entendu, l'enseignant les aidera dans cette tâche sûrement nouvelle pour beaucoup d'entre eux. L'enseignant devra ensuite procéder à l'élaboration d'une exploitation pédagogique de cette contextualisation en veillant toujours à préserver l'authenticité du contexte. Ceci ne peut être que facilité par l'emploi de documents authentiques.

Nous voulons montrer que l'approche essentiellement fonctionnelle de l'enseignement des langues de spécialité est bien entendu nécessaire, mais ne doit pas être au détriment de la dimension culturelle de l'enseignement d'une langue étrangère. 
17 Nous savons tous, pour avoir fait de la recherche pédagogique nous-mêmes, le fossé qui existe souvent entre la recherche (même appliquée) et la pratique, entre le théorique et le terrain, sans parler de recherche fondamentale!

\section{Le contexte culturel} identifiable. C'est peut-être parfois le cas, mais souvent celui-ci est confus, mal défini, sous-entendu par un ensemble d'implicites, La culture au sens anthropologique du terme étant souvent un ensemble de comportements, d'attitudes, etc., inconscientes dont l'apprenant est plus ou moins exclu. Mais c'est bien entendu à l'enseignant de l'élucider ou même de mettre en lumière ce qui n'est pas explicite.

Un contexte culturel peut également se présenter de manière diversifiée, où plusieurs contextes sont imbriqués, rendant perplexes les étudiants tout autant que l'enseignant, à la différence que celui-ci est sensé savoir pourquoi il est perplexe... Je ne fais pas référence ici à un contexte multiculturel qui va de soi dans une situation d'échange linguistique, mais plutôt à la juxtaposition de contextes différents, contrastés, voire opposés.

C'est souvent le cas en sciences économiques, où macro et micro peuvent s'enseigner en tant que sciences connexes, où une approche synchronique peut mener à une approche diachronique ou vice versa, ou encore la présentation de tel fait économique peut avoir comme arrière-plan une théorie économique. Toute décision économique est maintenant indissociable de l'évolution de la globalisation des marchés, en plus d'être étroitement liée à des considérations politiques ainsi qu'à des facteurs sociaux. Ce domaine a un système de référence si vaste qu'il est parfois difficile de définir un contexte, car pour le définir, il faut l'isoler, dans quel cas on risque de perdre " the total environment of the text ", pour reprendre à nouveau la définition de Halliday.

21 L'enseignant devra donc sélectionner judicieusement les éléments culturels et pluriculturels, sans pour autant les appauvrir et en veillant à ce que la spécificité du document à étudier prenne toute sa signification, par rapport à ces différents contextes. C'est l'aspect multifactoriel du référentiel qui donnera toute sa signification au texte, audelà du simple sens. Pour les étudiants, cette approche ne peut que rendre plus vivante l'étude d'un document spécialisé.

Un autre cas, plus rare, tout au moins dans un contexte pédagogique, est celui d'un contexte culturel que j'appellerai inversé. J'ai pris un exemple précis tiré de «RoyceLytton Foods» de la série vidéo Business Assignments. On y voit un homme d'affaires britannique en voyage au Koweït en train de négocier avec un cadre d'une société de ce pays, auquel il essaie de vendre ses produits. Ce qui est intéressant, c'est le retournement de situation par rapport aux clichés, aux stéréotypes auxquels on pourrait s'attendre. En fait, le Koweïtien connaît très bien la culture anglaise, au point de déstabiliser son interlocuteur en faisant référence, à propos de la région d'implantation de la société anglaise, à « Constable country ». Le Britannique ne saisit pas immédiatement l'allusion, hésite et perd encore un point dans le jeu qui est en fait un rapport de force dissimulé. II hésite car il est surpris par le côté culturellement hors contexte de cette remarque et non par manque de connaissance de sa propre culture. Je cite : 
Sheikh Sahid: And, where in England do you come from, Mr Rutherford?

Mr Rutherford: The company is based at Hadleigh, in Suffolk. It's on the eastern side of England.

Sheik Sahid: Yes, I know Sudbury very well. Magnificent countryside - Constable country?

Mr Rutherford: Constable? Oh, yes. Yes. Well, anyway, our position is that we'd like to...

Une telle allusion ne constitue pas à elle seule un contexte culturel. C'est l'ensemble de l'aspect référentiel du texte qui le construira.

Beaucoup d'autres connotations culturelles, implicites, sous-entendus et allusions peuvent être exploités et c'est une bonne occasion pour l'enseignant d'éviter les stéréotypes et clichés si appauvrissants pour les cours de langue. Dans un article, Louis Porcher (1982) reprend certaines idées de François Mariet :

Monsieur Mariet a raison, alors, de proposer que l'anthropologie, considérée comme résultante rigoureuse des diverses sciences humaines devienne la source rationnelle d'un véritable enseignement de la civilisation. Pour éviter la folklorisation, seule la contextualisation, c'est-à-dire l'inscription des divers phénomènes culturels dans le canevas d'ensemble qui leur donne leur cadre et permet de les comprendre réellement, permet de construire une véritable systématique de la civilisation étudiée. Économie, démographie, sociologie, politologie, ethnographie, histoire, géographie, esthétique font, entre autres, partie de cette panoplie.

Cette contextualisation me parait être la réponse à bien des interrogations que se posent les enseignants en langue de spécialité, tout au moins en ce qui concerne l'aspect culturel d'un enseignement à vocation scientifique et technique.

Avant de conclure, j'aimerais essayer de généraliser, en simplifiant peut-être à l'extrême l'approche didactique d'un document, pédagogique dans le premier cas, authentique dans le deuxième. Le fait qu'un document soit authentique ne constitue pas en soi une garantie d'efficacité pédagogique. Nous avons vu combien peu satisfaisant peut être le contexte, ou comme il peut prêter à confusion.

Je distinguerai donc deux stratégies. Dans le premier cas, il s'agit d'un document pédagogique sans contexte culturel. C'est donc à l'enseignant de procéder à une mise en contexte. Il va sans dire que, à moins que l'enseignant soit également un professionnel du domaine qu'il enseigne, ou tout au moins qu'il le connaisse très bien, imaginer un contexte professionnel sera une tâche très difficile.

Dans le deuxième cas, nous partons d'un document authentique. S'il n'est pas accompagné d'un contexte clairement défini (ce qui est assez probable), on ne peut que supposer que ce contexte de culture d'entreprise existe. Ce sera une fois de plus à l'enseignant de le créer, ou de le recréer, c'est-à-dire procéder au traitement didactique de ce document professionnel. Tâche également délicate, car il n'existe pas, pour autant que je sache, d'aide pédagogique à la didactisation de tels documents.

que les nouvelles technologies (professeur branché sur Internet, par exemple) peuvent nous rendre de précieux services, par un accès plus aisé et plus rapide à des informations utiles. N'oublions pas qu'il s'agit de préparations de cours et qu'elles doivent se faire en un temps raisonnable pour chacun d'entre nous.

Mais la solution de facilité, en anglais économique tout au moins, est de faire trop confiance à l'article de presse, généraliste ou même, disons, semi-spécialisée. Je ne m'exclus point de cette observation. En plus du risque de constituer un ensemble 
hétéroclite, je me permets de remettre en cause, dans le cadre de nos besoins pédagogiques, la pauvreté ou même l'absence de contexte dans ce type de publication, ceci pour des raisons commerciales évidentes. L'article est commode à exploiter mais il ne répond pas nécessairement à nos ambitions pédagogiques. Tournons-nous plutôt vers des revues scientifiques, en principe disponibles dans les salles de périodiques de nos bibliothèques universitaires.

\section{Conclusion} en charge des actes illocutoires (dans le sens défini par John Austin [1962]). En effet, j'ai observé dans mes propres cours qu'un des obstacles les plus redoutables est de mettre une intention dans ce qu'ils disent, et non pas se contenter de dire. Ceci peut les amener à une véritable réflexion métacognitive sur le discours, et notamment sur ce que leur interlocuteur veut dire, sur son intention dans le discours. Ceci me paraît fondamental à la prise de conscience de ce qu'est un véritable dialogue.

37 Mais n'oublions pas que le cours de langue est lui aussi un contexte situationnel qui doit être défini et sur lequel les étudiants doivent être amenés à réfléchir. Ils doivent être plus conscients du rôle du contexte culturel, qui est en fait un lien entre le cours de langue et le monde professionnel, entre l'apprenant et l'utilisateur. 
voudrais faire une dernière remarque sur la dichotomie compétence-performance d'après mes réflexions et mes investigations, je suis maintenant persuadé que la culture ne se mesure pas. Il n'est donc pas possible d'affirmer qu'elle améliore la compétence linguistique. En revanche, une compétence culturelle peut améliorer la performance linguistique, notamment en ce qui concerne l'acquisition du lexique, primordial en langue de spécialité.

\section{BIBLIOGRAPHIE}

Armengaud F. 1985. La Pragmatique. Que Sais-Je? n²230. Paris : Presses universitaires de France.

Austin, J.L. 1962. How to Do Things with Words. Oxford : Oxford University Press.

Baylon C. 1991. Sociolinguistique, Société, Langue et Discours. Paris : Nathan.

Baylon C. et X. Mignot. 1994. La Communication. Paris : Nathan Université.

Cummins J.P. 1983. « Language proficiency and academic achievement». In Oller, J.W. (dir.), Issues in Language Testing Research. Rowley, MA : Newbury House, 108-126.

Halliday M.A.K. 1978. Language as Social Semiotic. The Social Interpretation of Language and Meaning. Londres : Arnold.

Kramsch C. 1993. Context and Culture in Language Teaching. Oxford : Oxford University Press.

Miller T. 1994. « Culture and grammar in scientific English». ASp 5-6, 31-44.

Porcher L. 1982. « L'Enseignement de la civilisation en question ». Études de linguistique appliquée 47, 39-49.

Swales, J.M. 1990. Genre Analysis: English in Academic and Research Settings. Cambridge : Cambridge University Press.

Trocmé-Fabre, H. 1987. J'apprends donc je suis : introduction à la neuropédagogie. Paris : Les Éditions d'Organisation.

INDEX

Mots-clés : acte de parole, anglais de spécialité, contexte culturel, culture, performance, pragmatique, sociolinguistique

Keywords : cultural context, ESP, pragmatics, sociolinguistics, speech act

\section{AUTEUR}

\section{OLIVIER STURGE-MOORE}

Olivier Sturge-Moore enseigne l'anglais économique à l'Université Paris 1 Panthéon-Sorbonne depuis 1982. 